\title{
Amendment to: populations in environments with a soft carrying capacity are eventually extinct
}

\author{
Peter Jagers ${ }^{1}(1) \cdot$ Sergei Zuyev $^{1}$ (]) \\ Received: 10 October 2020 / Revised: 27 April 2021 / Accepted: 26 May 2021 / \\ Published online: 21 June 2021 \\ (c) The Author(s) 2021
}

\begin{abstract}
This sharpens the result in the paper Jagers and Zuyev (J Math Biol 81:845-851, 2020): consider a population changing at discrete (but arbitrary and possibly random) time points, the conditional expected change, given the complete past population history being negative, whenever population size exceeds a carrying capacity. Further assume that there is an $\epsilon>0$ such that the conditional probability of a population decrease at the next step, given the past, always exceeds $\epsilon$ if the population is not extinct but smaller than the carrying capacity. Then the population must die out.
\end{abstract}

Keywords Population dynamics $\cdot$ Extinction $\cdot$ Martingales $\cdot$ Stochastic stability

Mathematics Subject Classification 92D25 · 60G42 · 60K40

\section{Three assumptions and one result}

Denote population sizes, starting at time $\tau_{0}=0$, by $Z_{0}$, changing into $Z_{1}, Z_{2}, \ldots \in \mathbb{N}$ at subsequent time points $0<\tau_{1}<\tau_{2} \ldots$ Here $\mathbb{N}$ is the set of non-negative integers, and we make no assumptions about the times between changes. Let $\mathscr{F}_{n}$ be the sigmaalgebra of all events up to and including the $n$-th change - i.e. really all events, not only population size changes - and introduce a carrying capacity $K>0$, the population size where reproduction turns conditionally subcritical. More precisely:

Peter Jagers

jagers@chalmers.se

Sergei Zuyev

sergei.zuyev@chalmers.se

http://www.math.chalmers.se/ sergei

1 Department of Mathematical Sciences, Chalmers University of Technology and University of Gothenburg, SE-412 96 Gothenburg, Sweden 


\section{Assumption 1}

$$
\mathbb{E}\left[Z_{n+1} \mid \mathscr{F}_{n}\right] \leq Z_{n}, \quad \text { if } Z_{n} \geq K
$$

Further,

Assumption 2 There is no resurrection or immigration but, otherwise, a change is a change in population size:

$$
\begin{aligned}
& Z_{n}=0 \Rightarrow Z_{n+1}=0, \\
& Z_{n}>0 \Rightarrow Z_{n+1} \neq Z_{n} .
\end{aligned}
$$

Assumption 3 Non-extinct populations, smaller than the carrying capacity, run a definite risk of decreasing:

$$
\exists \epsilon>0 ; \forall n \in \mathbb{N}, 0<Z_{n}<K \Rightarrow \mathbb{P}\left(0 \leq Z_{n+1}<Z_{n} \mid \mathscr{F}_{n}\right] \geq \epsilon .
$$

Then:

Theorem 1 Under the three assumptions given, the population must die out: with probability $1, Z_{n}=0$ eventually.

The original paper (Jagers and Zuyev 2020) had a stronger third assumption, viz. that, whatever the population history, there must be a definite, strictly positive risk that the population size decreases by exactly one unit at the next change. This is not unnatural and can be interpreted as a possibility that a change involves no reproduction but merely the death of one individual. But it turns out to be unnecessary.

\section{The proof}

Like the original proof, this starts from stopping times $v_{1}, v_{2}, \ldots$ and $\mu_{1}, \mu_{2}, \ldots$, the former denoting the times of successive visits to the integer interval $[0, K)$, the latter the subsequent first hittings of levels $\geq K$. More precisely,

$$
v_{1}:=\inf \left\{n \in \mathbb{N} ; Z_{n}<K\right\},
$$

and for $k=1,2, \ldots$,

$\mu_{k}:=\inf \left\{n \in \mathbb{N} ; n>v_{k}\right.$ and $\left.Z_{n} \geq K\right\}, v_{k+1}:=\inf \left\{n \in \mathbb{N} ; n>\mu_{k}\right.$ and $\left.Z_{n}<K\right\}$.

As was noted, $v_{1}<\infty$, whereas the $\mu_{k}$ constitute an increasing sequence, possibly hitting infinity. Clearly, $v_{k}<\infty, \mu_{k}=\infty$ means that the population dies out at or after $v_{k}$, without ever reaching $K$ again. Also for any $k, \mu_{k}<\infty \Rightarrow v_{k+1}<\infty$. Proceeding like in the original paper, note that

$$
Z_{n} \rightarrow 0 \Leftrightarrow \exists n \in \mathbb{N} ; Z_{n}=0 \Leftrightarrow \exists k ; \mu_{k}=\infty,
$$


and

$$
\mathbb{P}\left(\exists k ; \mu_{k}=\infty\right)=\lim _{k \rightarrow \infty} \mathbb{P}\left(\mu_{k}=\infty\right)=1-\lim _{k \rightarrow \infty} \mathbb{P}\left(\mu_{k}<\infty\right)
$$

But

$$
\mathbb{P}\left(\mu_{k}<\infty\right)=\mathbb{P}\left(\mu_{k}<\infty, v_{k}<\infty\right)=\mathbb{E}\left[\mathbb{P}\left(\mu_{k}<\infty \mid \mathscr{F}_{v_{k}}\right) ; v_{k}<\infty .\right]
$$

For short, write

$$
D_{n}:=\left\{Z_{n} \leq\left(Z_{n-1}-1\right)^{+}\right\}
$$

for the event that the $n$-th change is a decrease, provided $Z_{n-1}>0$ (and of course the population remains extinct if $Z_{n-1}=0$ ). By Assumption 3, $Z_{n}<K$ implies that

$$
\begin{aligned}
\mathbb{P}\left(\cap_{j=1}^{K} D_{n+j} \mid \mathscr{F}_{n}\right) & =\mathbb{E}\left[\mathbb{P}\left(D_{n+K}\left|\mathscr{F}_{n+K-1} ; \cap_{j=1}^{K-1} D_{n+j}\right| \mathscr{F}_{n}\right]\right. \\
& \geq \epsilon \mathbb{P}\left(\cap_{j=1}^{K-1} D_{n+j} \mid \mathscr{F}_{n}\right) \geq \ldots \geq \epsilon^{K} .
\end{aligned}
$$

Since $Z_{n}<K$ implies that $Z_{n+K}=0$ on the set

$$
\cap_{j=1}^{K} D_{n+j},
$$

and the population size never crosses the carrying capacity, we can conclude that

$$
\begin{aligned}
\mathbb{P}\left(\mu_{k}=\infty\right) & =1-\mathbb{P}\left(\mu_{k}<\infty\right) \\
& \geq 1-\left(1-\epsilon^{K}\right) \mathbb{P}\left(\mu_{k-1}<\infty\right) \geq \ldots \geq 1-\left(1-\epsilon^{K}\right)^{k} \rightarrow 1 .
\end{aligned}
$$

The theorem follows.

\section{Reference}

Jagers P, Zuyev S (2020) Populations in environments with a soft carrying capacity are entually extinct. J Math Biol 81(3):845-851. https://doi.org/10.1007/s00285-020-01527-5

Publisher's Note Springer Nature remains neutral with regard to jurisdictional claims in published maps and institutional affiliations. 